\title{
I have to go online next week?! Practical suggestions based on Ke (2016)
}

\author{
Alexander Nagurney ${ }^{1}$ \\ Accepted: 31 October 2020 / Published online: 10 November 2020 \\ (c) Association for Educational Communications and Technology 2020
}

\begin{abstract}
Activation of known material, the importance of context and narrative, opportunities for reflection, and appropriate feedback are all major issues to be taken into consideration when designing online courses. The purpose of this review is to discuss the ways in which these various themes, elucidated by $\mathrm{Ke}$ (Designing and integrating purposeful learning in game play: A systematic review. Educational Technology Research and Development, 64, 219-244. https://doi.org/10.1007/s11423-015-9418-1, 2016), can be brought to life when transitioning a course to an online format. Issues related to this review will be discussed from a practical perspective aimed at assisting course instructors when making a fast transition to an online teaching modality.
\end{abstract}

Keywords Knowledge activation $\cdot$ Context $\cdot$ Narrative $\cdot$ Reflection $\cdot$ Feedback

Online course delivery has become an increasingly prominent mode of education over the past several years and has taken on special prominence recently as the coronavirus has pushed more and more universities to move to either a largely or completely online modality. Ke (2016) provides some important themes that instructors can utilize when designing online courses. While this article focused specifically on the role that gaming plays in the educational process, my plan is to extrapolate larger "back-to-basics" lessons than can be applied to the online learning process, particularly in a situation in which a fast transition to an online course is required. The focus will be on how these lessons learned from the gaming perspective can be applied through a gamification lens, thus bridging the two areas together. The reason for this shift in focus is that the principles of gamification can often be more readily applied than those of gaming and this is an important consideration when a quick shift to an online modality becomes necessary. Issues related to this review will therefore be discussed from a practical perspective aimed at assisting course instructors when making a fast transition to an online teaching modality.

Ke (2016) points out that gaming can be used as a method to activate and explore previously-learned knowledge, particularly in games that provide a virtual world simulation experience. This is a crucial area for learning in that activation of previous knowledge sets

Alexander Nagurney

nagurney@ hawaii.edu

1 Department of Psychology, University of Hawaii at Hilo, 200 W. Kawili St, Hilo, HI 96720, USA 
the scene for new learning. In a practical sense, courses should make it a point to at least briefly review both prerequisite material as well as newly-acquired material. These reviews could be as simple as recognition activities that prime the material for students, such as multiple choice or true/false assessments. Once the material is activated, new learning can occur. Much like Ke discussed the idea of the importance of the simulating the world, a gamified course could include activities such as scavenger hunts that require students to leave the online classroom and identify themes learned in previous courses in either the real-world setting or on other websites beyond the classroom.

A second major theme articulated by Ke (2016) is that learning is most useful if it represents operations from a real world context. Such a context makes the learning environment more intrinsically motivating, thus more engaging to students. More engagement with the material should be associated with more learning. Much like the fantasy-based role playing games discussed by Ke, courses could include working with real world problems that are presented to students who are in turn asked to act as consultants to help solve the problems by utilizing theories learned in class. The act of having students consider solutions to such problems should make the material seem more tangible to students, thus increasing their intrinsic motivation and their learning. In keeping with the gamification theme, students could be rewarded or earn points for the consultation assistance they provide to their classmates.

A third theme identified by $\mathrm{Ke}$ (2016) is essentially a combination of the first two themes and can be summarized by the idea that a narrative is an important facet of learning. A narrative can be defined as a story that is being told through the course content. It begins with background information (as can be seen in the first theme concerning knowledge activation), continues with addressing learning by using newly acquired information to solve new problems (as can be seen with the second theme concerning the importance of context), and closes by addressing the importance of possible future outcomes. This final component is possibly the most important step here because knowing where a problem or an area is moving in the future requires a firm understanding of where it's been in the past and where it currently stands. On a practical level, students might be asked to have a discussion concerning where their field is going or what the most important issues will be in the future for their field.

Ke (2016) points out a fourth theme, the importance of reflection in the learning process. He discusses the idea of "learning moments" in games during which players are required to consciously recall past experiences and prior knowledge and how this information connects to the current game scenario. This relates to the gamified approached in that students often face the problem that they may be taking several courses in a number of different fields all at the same time. I believe that the most important form of reflection is allowing students to step back from the course material and consider the "big picture" of their educational experiences. They may be asked to write a reflection that requires them to find themes across their courses, to consider how what they're learning in their current courses relates to their previous classwork, and how their current courses have made them better learners or helped them to make important discoveries about the self. On an anecdotal level, I can report that I use reflection assignments such as these in my own courses and students have often reported that these assignments have been especially useful and insightful for them.

The fifth and final theme articulated by Ke (2016) is that scaffolding and cues can be essential to the learning process. In games this information might be presented as a rough map that gives students a general area about where to go next, but leaves it to the student to find the more exact routing. This scaffolding is often accomplished in coursework through 
the use of instructor comments on rough drafts, the use of feedback from the instructor or classmates in online forum discussions, or perhaps class emails from the instructor that summarize general problem areas during the previous week without targeting specific students. All of these methods for providing feedback have some value, however they should be used cautiously. To make a very general statement, I believe that part of the educational experience is a student learning what they don't know. This isn't necessarily an instructor simply giving the student the right answer, but it's rather giving the student just enough information that the student will know where to look to find the information they need. In other words, learning, while assisted as needed, should remain largely autonomous. This balance of giving information versus giving cues to find the information is one that needs to be carefully struck by educators.

In closing, the themes identified by Ke (2016), while largely aimed at the role of gaming in the educational process, do provide a good starting point for instructors when thinking about the types of assignments to give students and the role of instructor feedback in those assignments, particularly in the area of gamification. Activation of known material, the importance of context and narrative, opportunities for reflection, and appropriate feedback are all major issues to be taken into consideration when designing online courses. Taking these issues into account should make for positive learning experiences for students and instructors alike.

\section{Compliance with ethical standards}

Conflict of interest The author has no conflicts of interests to disclose.

Informed consent There was no data collected as part of this review paper beyond what was already reported in $\mathrm{Ke}$ (2016), therefore informed consent and compliance with the ethical standards involving human participants is assumed to have been received by the original authors of the articles reviewed by Ke (2016).

\section{Reference}

Ke, F. (2016). Designing and integrating purposeful learning in game play: A systematic review. Educational Technology Research and Development, 64, 219-244. https://doi.org/10.1007/s1142 3-015-9418-1.

Publisher's Note Springer Nature remains neutral with regard to jurisdictional claims in published maps and institutional affiliations.

Alexander Nagurney is an Assistant Professor of Psychology at the University of Hawaii at Hilo. His teaching interests include social psychology, interpersonal relationships, personality, and statistics and research methods. Dr. Nagurney conducts research in the areas of how interpersonal relationships influence health outcomes as well as the ways in which people think about infidelity in relationships. When not teaching and researching, he loves to travel to pretty much anywhere in the world (mostly to try the food). 